\title{
EMPIRISMO, ESTRUCTURALISMO Y CAMBIO CIENTÍFICO
}

\author{
SUSANA LUCERO \\ Universidad de Buenos Aires
}

\begin{abstract}
In the last decades there was a structural turn in the classic debate between scientific realists and antirealists with empiricist orientation. Two main arguments support the realist conception: the 'No Miracle Argument' and the thesis of continuity. The thesis of continuity states that some parts of a theory are retained when a scientific change takes place. In a current famous article, J. Worrall (1989) defends the continuity argument by stating that what is preserved in the succession of two empirically successful theories are the relations among the postulated entities and not the nature of the relata (structural or syntactical realism). Based on this perspective, van Fraassen introduces his position named Empiricist Structuralism, which claims that only the structures of phenomena are retained. This conception tries to explain the success of science and at the same time defend the continuity of structures. I will demonstrate in this paper that the accomplishment of the two mentioned requirements imply a capitulation in favor of realistic intuitions.
\end{abstract}

El clásico debate filosófico entre realistas científicos y anti-realistas de orientación empirista ha tomado, en los últimos años, un nuevo sesgo conforme al desarrollo experimentado por la ciencia misma; este sesgo puede caracterizarse como el predominio de un enfoque estructural. Los realistas científicos tradicionales continúan defendiendo una forma robusta de realismo científico epistémico e intentan dar sostén a sus puntos de vista a través de varios argumentos entre los cuales sobresale el argumento del no milagro. Expresado de manera sintética, el argumento sostiene que el éxito empírico de las teorías científicas sería un milagro, una especie de coincidencia cósmica, a menos que se postule la verdad o aproximación a la verdad de aquellos principios que permitieron inferir las consecuencias empíricas exitosas. Algunos autores opinan que los anti-realistas — sean instrumentalistas o de orientación pragmática-, no pueden ofrecer una respuesta convincente del éxito de la ciencia; la ventaja del realismo radica justamente en ser la única concepción que no hace del éxito científico un milagro.

Estrechamente vinculado a este argumento, otra tesis conexa aparece como un importante pilar de las intuiciones realistas, a saber la idea de que en el cambio

Principia, 12(1) (2008), pp. 87-96. Published by NEL — Epistemology and Logic Research Group, Federal University of Santa Catarina (UFSC), Brazil. 
científico de una teoría pasada exitosa $T$ a su sucesora exitosa $T^{\prime}$, hay algo que se conserva o retiene de modo que el cambio científico es esencialmente acumulativo. La dependencia del realismo científico de la idea de continuidad, aun en los casos de revolución o cambio radical, parece ser un hecho indiscutible. No podría ser de otra manera, ya que si dos teorías sucesivas fueran teóricamente incompatibles entre sí y no tuvieran elementos en común, el realista no podría afirmar que la primera es aproximadamente verdadera y que también lo es su sucesora, so pena de caer en inconsistencia.

La piedra de toque que hoy parece distanciar a partidarios del realismo científico radica en la especificación de qué exactamente es lo que se mantiene a través del cambio. Para un realista científico tradicional, lo que permanece a través del cambio es el contenido empírico corroborado de la antigua teoría más los procesos y mecanismos causales, de carácter teórico (inclusive los supuestos auxiliares) que intervinieron en la derivación de las consecuencias empíricas exitosas. Últimamente las posiciones conservadoras adhieren al principio de divide et impera considerando que no todo lo que una teoría postula está justificado; luego es racional creer sólo en aquellas partes de la teoría acerca de las cuales estamos garantizados. (Psillos 1999, p. 108)

En 1989, John Worrall presentó una variante debilitada de esta concepción, la cual intenta recoger, en particular, los hallazgos de la historia de la ciencia. Éstos muestran el desarrollo científico como una sucesión de teorías refutadas, lo que permite derivar inductivamente la conclusión de que las teorías contemporáneas más exitosas correrán la misma suerte. Este argumento es ampliamente conocido como argumento de la meta inducción desastrosa.(Laudan 1997). Worrall afirma haber rescatado las intuiciones básicas implicadas en ambos argumentos, la metainducción desastrosa y el argumento del no milagro, reuniendo así lo mejor de los dos mundos.

Para Worrall, lo que precisamente se pierde en el pasaje de una teoría antigua a su sucesora es el contenido o interpretación teórica. Lo que permanece, además de las consecuencias empíricas correctas, es la "forma" o "estructura" de la teoría antigua expresada en las ecuaciones matemáticas; en tanto que las propiedades cualitativas de los relata que soportan las relaciones permanecen desconocidas. Estas restricciones constituyen el núcleo del realismo sintáctico o estructural. La propuesta es superadora del realismo tradicional en la medida en que no avala la continuidad del contenido teórico. Tomando como ejemplo la teoría óptica de Fresnel, demuestra que su éxito empírico se debió a que capturó correctamente la estructura de los fenómenos luminosos. La teoría atribuyó a la luz movimientos en un medio sólido elástico, el éter, y fue reemplazada posteriormente por

Principia, 12(1) (2008), pp. 87-96. 
la óptica de Maxwell, la cual afirma que el desplazamiento lumínico se debe a vibraciones en un campo electromagnético. Está claro que ambas teorías realizan postulaciones ontológicas opuestas con respecto a la naturaleza de la luz. De todos modos, es imposible negar que: (a) la teoría de Fresnel, aunque falsa, fue empíricamente exitosa ya que formuló predicciones corroboradas sorprendentes; (b) su éxito empírico no es milagroso, pues "... no es un milagro que su teoría tuviera el éxito empírico predictivo que tuvo; no es un milagro porque la teoría de Fresnel, como la ciencia lo demostró más tarde, atribuyó a la luz la "estructura" correcta". (Worrall 1997, p. 157).

Ahora bien, iqué aspectos del realismo estructural lo acreditan como una concepción auténticamente realista? A propósito de este problema, debe subrayarse que una tesis central del realismo científico es la presuposición de que existe una realidad extra, por debajo o más allá del mundo fenoménico, respecto de la cual las teorías intentan ofrecer una descripción literalmente verdadera o aproximadamente verdadera, postulando procesos o mecanismos causalmente responsables de las apariencias. Algunos indicios de esta sospecha realista se insinúan en el artículo de Worrall, si bien es cierto, estos pronunciamientos no son explícitos. En primer lugar, destacamos la omnipresente referencia a Poincaré como el filósofo que anticipó la vía por la cual las teorías llegan a rastrear ciertos aspectos del mundo real en un nivel no observable. Para Poincaré, las teorías no son simples recetas prácticas, "sus ecuaciones expresan relaciones y si las ecuaciones permanecen verdaderas, es que las relaciones preservan su realidad. [...] Las relaciones verdaderas entre estos objetos reales son la única realidad que podemos alcanzar. (Poincaré, H. La ciencia y la hipótesis, citado por Worrall 1997, p. 158). Las cursivas me pertenecen). Worrall enfatiza que estas afirmaciones no podrían corresponder a un instrumentalista, rótulo que se le viene atribuyendo al filósofo francés erróneamente.

En segundo lugar, hacia el final del artículo, se menciona la mecánica cuántica. Worrall suscribe una visión realista de los estados cuánticos aunque su interpretación no es coincidente con la de Einstein. La teoría cuántica "parece haber capturado la estructura real del universo; el estado cuántico de un sistema realmente evoluciona y cambia como lo describe la mecánica cuántica" (Worrall 1997, p. 162). Y más adelante agrega:

El realista estructural simplemente sostiene que, en vistas del enorme éxito empírico de la teoría, la estructura del universo (probablemente) sea algo así como lo mecánico cuántico. (Worrall 1997, p. 163)

Estas declaraciones no dejan dudas acerca de las intuiciones que subyacen en Principia, 12(1) (2008), pp. 87-96. 
la propuesta de Worrall. La formulación del argumento del no milagro y la tesis de que, a través del cambio científico, algo del nivel de lo no observable realmente se conserva y no solamente los éxitos empíricos, constituyen conjuntamente bases suficientes para atribuir una forma de realismo científico epistémico — si bien débil- a la concepción que Worrall nos ha presentado.

\section{2}

En su reciente artículo "Structure: its Shadow and Substance" (2006), Van Fraassen se incorpora a la discusión acerca del enfoque estructuralista en los debates entre el realismo científico y el empirismo constructivo. Tras haber delineado la evolución histórica desde el nacimiento de la ciencia moderna en el siglo XVII, exalta la circunstancia de que las teorías fácticas han protagonizado un proceso de creciente matematización, con el consiguiente predominio de las estructuras matemáticas. "Reificación" y "Estructuralismo" constituyeron una suerte de mecanismos defensivos contra la actitud realista ingenua. Cada una de estas estrategias intentó articular una imagen científica acorde con los desarrollos de la ciencia misma. El final del siglo XIX, y más aún el siglo XX, fueron testigos de la preeminencia del estructuralismo tanto entre los científicos como entre los filósofos de la ciencia. En el nivel meta-científico, la propuesta de John Worrall representa un claro ejemplo.

A pesar de la declarada intención de hacer justicia a las demandas originales de Worrall, el realismo estructural resulta, en este artículo, objeto de las más severas críticas. Van Fraassen juzga "esquizofrénica" la explicación del éxito de la ciencia brindada por Worrall; respecto de la propuesta de este autor, reconoce dos hechos:

(i) La ciencia exhibe un éxito empírico indiscutible y evoluciona en el sentido del progreso. El éxito se identifica con la formulación de predicciones empíricamente correctas.

(ii) Hay continuidad a través del cambio teórico, es decir una parte de la antigua teoría exitosa es retenida en la nueva, aun en los casos de cambio radical o revolucionario.

Worrall está acertado al defender (i) pero se equivoca respecto de (ii) porque duplica innecesariamente lo retenido en el nivel formal. No solamente apuesta a la conservación de la estructura de los fenómenos — algo con lo cual los empiristas estarían en total acuerdo- sino también a la que informa las entidades inobservables: "El conocimiento acumulado es acerca de aspectos del mundo que

Principia, 12(1) (2008), pp. 87-96. 
trascienden la realidad empírica, desplegada en observación y experimentación, aspectos de la realidad (que yacen) detrás de los fenómenos" (van Fraassen 2006, p. 295). En esta duplicación descubre van Fraassen el nudo de la esquizofrenia, pero la duplicación es innecesaria pues una versión alternativa del estructuralismo está a nuestro alcance. La visión propuesta ahora es denominada estructuralismo empírico, la cual sostiene que "hay efectivamente algo que se preserva en el cambio de teorías, es la estructura de los fenómenos" expresada en ecuaciones matemáticas simples. Son ejemplos históricos de este tipo las leyes de Arquímedes, de la inercia, de la caída libre, del péndulo.

Además el estructuralismo empírico está en condiciones de ofrecer una versión empirista del argumento del no milagro exenta de pretensiones ontológicas, pues para dar fundamentos del cambio acumulativo no es necesario apostar a la existencia de procesos o entidades inobservables. Solamente dos clases de cosas son requeridas: los fenómenos, procesos y eventos observables, por una parte y las estructuras estudiadas por la matemática, por otra.

El éxito de la ciencia es explicado mediante la apelación a un principio débil que van Fraassen denomina el requisito de sucesión: "una teoría nueva está relacionada con su predecesora de tal manera que podemos explicar el éxito empírico de la vieja teoría si aceptamos la nueva" (van Fraassen 2006, p. 298).

Ahora bien, una candidata a la sucesión "real" tiene que satisfacer ciertos requerimientos: la nueva teoría debe duplicar el éxito empírico de su predecesora realizando predicciones más precisas en el mismo dominio de fenómenos en que había acertado la anterior, pero además tiene que explicar cómo y por qué su antecesora tuvo el éxito que tuvo. Otras credenciales para su candidatura son, por supuesto, la capacidad de realizar nuevas predicciones, de dar cuenta de los fallos de la teoría anterior y de reestructurar los conceptos antiguos de una forma novedosa. Así pues, el estructuralismo empírico parece haber logrado su doble objetivo: explicar el éxito empírico de la ciencia y simultáneamente defender la conservación de la estructura a través del cambio científico. Mostraremos que el cumplimiento de estas demandas implica, en cierto modo, una capitulación a favor de intuiciones que son y han sido típicamente características del realismo científico.

3

El estructuralismo empírico, al parecer, ha copiado demasiadas cosas del realismo científico. De la concepción de Worrall incorporó la intuición de que lo que permanece a través de los cambios es la estructura; del realismo científico en general se apropió del argumento del no milagro. Notemos, en primer lugar, que van

Principia, 12(1) (2008), pp. 87-96. 
Fraassen destaca correctamente las dificultades asociados a la noción de estructura. En efecto, la tan renombrada dicotomía estructura-contenido es aplicada de manera ambigua tanto a los objetos de la naturaleza como a las partes de una teoría científica. Cuando se usa para referirse a nuestro conocimiento del mundo, los distintos autores se han visto conducidos a distinguir entre propiedades intrínsecas y relacionales, propiedades causales y de detección, etc. (Ckakravartty 2004), y a menudo han desembocado en disquisiciones metafísicas de dudosa precisión. De todos modos, creemos en la posibilidad de dejar la dicotomía como un concepto primitivo, con un valor pragmático, tal como ha ocurrido con tantas otras clasificaciones en filosofía de la ciencia. Otra opción es conservarla como una distinción no ontológica sino con un significado exclusivamente epistémico, lo cual incluye la ventaja de que nos permite separar las partes de una teoría sobre las que tenemos buenas razones para creer, de aquellas otras acerca de las cuales no tenemos garantizadas las creencias. La sugerencia de van Fraassen de que la dicotomía es contextualmente dependiente tiene completa cabida en esta propuesta.

Sea como fuere, en el marco de la concepción de Worrall, la postulación de la preservación de la estructura con respecto a mecanismos o procesos inobservables funciona bien porque deja de lado la naturaleza de los mentados procesos, en virtud de que el desarrollo de la ciencia va mostrando su caducidad. Se podría alegar que un realismo de este tipo es débil, que es una forma intermedia entre el realismo científico y el instrumentalismo: concedido, pero ciertamente la distinción trazada cumple una función en la explicación del cambio en el marco general de la teoría de Worrall. Resulta lícito preguntarse, entonces, icon qué fin se enfatiza la preservación de la estructura en el nivel de los fenómenos observables cuando en este segundo caso, aunque no en el primero, el contenido fenoménico es retenido junto con la estructura? La postulación del estructuralismo en el nivel de lo exclusivamente empírico parece trivial.

Queda todavía por resolver cuál es el status que van Fraassen les atribuye a las estructuras. El artículo que estamos analizando oscila entre la reificación y - valga la redundancia- el estructuralismo de las estructuras mismas. Así, en un párrafo se dice que hay dos clases de cosas: los fenómenos por una parte, y las estructuras matemáticas por la otra; y en otro lugar se sostiene que el éxito de las teorías pasadas radica en que los modelos o representaciones de los fenómenos correspondientes a tales teorías fueron parcial o aproximadamente exactos, ya que "hubo algo que ellos captaron correctamente: la estructura de esos fenómenos con un cierto nivel de aproximación" (van Fraassen 2006, p. 303). Estas afirmaciones sugieren que las estructuras tienen algún tipo de entidad, pero si

Principia, 12(1) (2008), pp. 87-96. 
esto es así, ellas no pertenecen seguramente a la clase de los fenómenos observables. Otra interpretación insinuada en el mismo ensayo es ver las estructuras como patrones de relaciones relevantes que pueden aplicarse a diferentes dominios de la naturaleza; en este segundo sentido adquieren el status de herramientas matemáticas que la ciencia construye para hacer inteligibles los fenómenos: "[... ] lo que es transparente a la mente es la estructura matemática que crea la ciencia teórica, y lo que adicionalmente es comprendido es cómo — de manera enteramente contingente y arracional-, esta estructura es aplicada a los fenómenos, dentro de nuestro alcance" (van Fraassen 2006, p. 287; las cursivas me pertenecen ). La idea de que las estructuras matemáticas son entidades creadas por la mente ha sido destacada también por J. Ladyman, a propósito del problema de la existencia de modalidades objetivas. ${ }^{1}$ La interpretación instrumentalista de las estructuras es más acorde con los supuestos antirrealistas defendidos por van Fraassen, pero vuelve completamente irrelevante la tesis enunciada en La imagen científica acerca de que la ciencia está construida en un lenguaje literal y que sus afirmaciones son auténticos enunciados capaces de soportar condiciones de verdad. (van Fraassen 1980, p. 10).

El segundo tema importante para nuestro objetivo se refiere al requisito de la sucesión de teorías y cómo éste podría satisfacer la intuición del no milagro. En efecto, van Fraassen sostiene:

La sucesión de la ciencia no es un milagro porque en todo cambio teórico tanto el éxito empírico pasado conservado como el nuevo éxito empírico son necesarios como credenciales para la aceptación. (van Fraassen 2006, p. 298-9)

Considero que el principio de la sucesión "real" no es tan débil como se sugiere ya que exige, como condición sine que non, que la candidata al trono (del éxito) explique cómo y por qué la teoría precedente tuvo el éxito que tuvo. Por ejemplo, la teoría especial de la relatividad puede explicar por qué las ecuaciones newtonianas funcionan tan bien en dominios donde los movimientos son comparativamente lentos (respecto de la velocidad de la luz) y en intervalos comparativamente cortos; es decir la nueva teoría puede explicar los éxitos empíricos alcanzados por la teoría superada, de modo que si se cumplen los requisitos mencionados, obtenemos una mejor comprensión del éxito de la ciencia. Ahora bien, si esto no constituye una explicación, entonces nada lo es. Al formular estas opiniones, van Fraassen está haciendo una concesión a las demandas explicativas, por más que las quiera investir de un ropaje empirista o que prefiera sustituir el término "explicación" por el de "demostración".

Principia, 12(1) (2008), pp. 87-96. 
Debe recordarse además que las detracciones a la explicación son frecuentes en casi todas sus obras; desde siempre van Fraassen ha insistido en que la búsqueda de explicaciones responde a un prejuicio metafísico, que ella no proporciona una información diferente de la que ofrece una descripción y que la capacidad explicativa de una teoría es una virtud ensalzada por los realistas científicos para quienes la explicación es "algo irreductible y especial" (van Fraassen 1980, p. 155). Las demandas de explicación son enlistadas con otro conjunto de convicciones generales arraigadas en un instinto metafísico al que pertenecen también la creencia en una lógica inductiva, en que la racionalidad consiste en seguir una regla y otras por el estilo. En contraste con la predicción, la explicación no aspira simplemente a "salvar los fenómenos" sino que intenta indagar las causas que los han producido, buceando en el reino de lo inobservable. Frente a este posicionamiento, el realista está en su derecho de atribuir a van Fraassen un prejuicio contrario, a saber: la creencia de que postular un estado de cosas que vaya más allá de lo dado directamente a la observación es pura metafísica, ipero acaso esta identificación no es ella misma una convicción general derivada de un instinto metafísico de dirección opuesta?

Vale la pena aclarar que el rechazo de las explicaciones no es una posición nueva en filosofía de la ciencia, la encontramos reflejada, por ejemplo, en los escritos de Pierre Duhem. Una teoría física, para Duhem, tiene que lograr la autonomía respecto de la metafísica, su función es ofrecer una representación y una clasificación de las leyes experimentales y no una explicación de los fenómenos. Su cometido es predecir; mientras que la tarea de explicar corresponde a la metafísica, pues "explicar", en términos de Duhem, no es otra cosa que "despojar la realidad de las apariencias que la envuelven como velos, a fin de contemplar esa realidad cara a cara" (Duhem 1914, p. 4). Está claro que este autor tiene en mente un concepto de explicación muy cercano al de Aristóteles, en el sentido de la búsqueda de esencias o naturalezas profundas que se ocultan bajo el velo de las apariencias. A más de 100 años de estos pronunciamientos, otras concepciones más naturalizadas de la explicación están a nuestro alcance, sin que por eso nos debamos sentir arrastrados hacia esa ciénaga metafísica que se traga a los investigadores en nombre de los verdaderos fundamentos del ser (van Fraassen 2006, p. 303). De todos modos, Van Fraassen no ha podido eludir la tentación de apropiarse de intuiciones que son típicamente características del realista.

Cabe concluir que la evolución del empirismo constructivo hacia el estructuralismo empírico no ha llevado agua para el molino empirista. Algunas concesiones hechas al argumento del no milagro y al realismo estructural son más bien capitulaciones a favor del realismo, o cuando menos simples recursos persuasivos

Principia, 12(1) (2008), pp. 87-96. 
para que, en el fondo, nada cambie. Una vez más, y a pesar del giro estructuralista operado, el resultado arroja "tablas" en este prolongado, controvertido y riquísimo debate entre realistas científicos y anti-realistas de cuño empirista.

\section{Referencias}

Duhem, P. [1906] 1914. La théorie physique, son object, sa structure. Paris: Marcel Rivière.

Chakravartty, A. 2004. Structuralism as a form of scientific realism. Internacional Studies in the Philosophy of Science 18(2-3): 151-71.

Ladyman, J. 2000. What's Really Wrong with Constructive Empiricism? Van Fraassen and the Metaphysics of Modality. Brit. J. Phil. Sci. 51: 837-56.

Laudan, L. [1981] 1997. A Confutation of Convergent Realism. In Papineau, D. (ed.) The Philosophy of Science. New York: Oxford University Press, p. 107-38.

Lucero, S. 2007. Los desafíos del realismo estructural. In Bobenrieth, A. (ed.) Ciencias Formales y Flosofía. Valparaíso: Edeval, p. 105-18.

Lucero, S. \& Orzeszko, R. 2006. Debates en torno al realismo estructural. Epistemología e Historia de la Ciencia. 12: 375-81.

Psillos, S. 1999. Scientific Realism: How science tracks truth. London: Routledge.

van Fraassen, B. C. [1980] 1985. The Scientific Image. New York: Oxford University Press. - 2002. The Empirical Stance. Yale University Press.

—. 2006. Structure: Its Shadow and Substance. Brit. J. Phil. Sci. 57: 275-307.

Worrall, J. [1989]1997.. Structural Realism: The Best of Both Worlds? In Papineau, D. (ed.) The Philosophy of Science. New York: Oxford University Press, p. 139-65.

\section{Keywords}

Scientific realism, antirealism, structural realism, constructive empiricism, empiricist structuralism.

Susana Lucero

Universidad de Buenos Aires

Dpto IPC. CBC.

Ciudad Universitaria

Buenos Aires

Argentina

lususa@arnet.com.ar

Principia, 12(1) (2008), pp. 87-96. 


\section{Resumo}

Nas últimas décadas houve uma virada estrutural no debate clássico entre realistas científicos e anti-realistas de orientação empirista. Dois argumentos centrais dão suporte à concepção realista: o argumento do não-milagre e a tese da continuidade. Essa tese sustenta que algumas partes de uma teoria são mantidas quando tem lugar uma mudança científica. Em um famoso artigo, J. Worrall (1989) defende o argumento da continuidade afirmando que aquilo que é preservado na sucessão de duas teorias empíricas bem-sucedidas são as relações entre as entidades postuladas e não a natureza dos relata (realismo estrutural ou sintático). Baseado em tal perspectiva, van Fraassen introduz sua posição, denominada estruturalismo empírico, cuja tese central é de que somente as estruturas dos fenômenos são mantidas. Essa concepção tenta explicar o sucesso da ciência e, ao mesmo tempo, defende a continuidade das estruturas. Demonstrarei neste artigo que o cumprimento dos dois requisitos mencionados implica em uma capitulação em favor de intuições realistas.

\section{Palavras-chave}

Realismo científico, anti-realismo, realismo estrutural, empirismo construtivo, estruturalismo empirista.

\section{Notas}

${ }^{1}$ Ladyman señala que para van Fraassen las modalidades tienen solamente un valor pragmático y son teóricamente dependientes; más precisamente dependen de los modelos incluidos en las teorías empíricamente adecuadas. Y agrega que "van Fraassen también parece pensar 'que los modelos mismos sólo existen en la mente y, en consecuencia, que el locus ontológico de la modalidad es nuestra conciencia y no el mundo que describimos"' (Ladyman 2000, p. 848-9; las cursivas me pertenecen). 